\title{
Low-dose Aspirin for Preventing Preterm Birth: A Meta-Analysis
}

\author{
Fuchuan Wang*, Wei Yi, Li Li, Mingfang Zhou, Xiuzhen Cao, Juanjuan Yao and Xuemei Liu \\ Resident, Department of Obstetrics and Gynecology, Beijing Di-Tan Hospital, Capital Medical University, China
}

\begin{abstract}
Objective: Preterm birth (PTB) remains a leading cause of neonatal mortality and is a disproportionately high burden. Aspirin is increasingly used to prevent PTB, so it is necessary to further evaluate the effect of different doses of aspirin in different gestational weeks on PTB.
\end{abstract}

Data Sources: A systematic literature search of the PubMed, Embase, Medline, ClinicalTrials.gov, and the Cochrane database (from database inception to 1 March 2020) for randomized controlled trials (RCTs) that examined treatment with aspirin versus placebo for PTB.

Methods of Study Selection: Selection criteria included studies evaluating the effect of aspirin compare with placebo on PTB. Using EndNote, three authors screened abstracts and two screened full articles for inclusion.

Main Results: Three authors extracted data from each article and analyzed the data using a descriptive approach. Thirty-one RCTs were included in the meta-analysis. Aspirin reduced the incidence of PTB $(p<0.00001$; risk ratio $(\mathrm{RR})=0.89)$. And then we do a stratified analysis. Low dose aspirin, commenced $\leq 13$ weeks gestation $(p=0.0006 ; \mathrm{RR}=0.83)$, commenced $\leq 20$ weeks gestation $(\mathrm{p}<0.00001 ; \mathrm{RR}=0.83)$, commenced $>13$ gestation weeks $(p=0.001 ; \mathrm{RR}=0.91),>20$ gestation weeks $(p=0.50 ; \mathrm{RR}=0.94)$, aspirin significantly reduces the incidence of PTB; however aspirin did not decrease the incidence of PTB if it commenced $>20$ gestation weeks. According to the different dose of aspirin we further stratified all studies, received 60mg aspirin ( $p=0.007 ; R R=0.92)$, received $75 \mathrm{mg}$ aspirin $(\mathrm{RR}=0.52, P=0.008)$, received about $80 \mathrm{mg}$ aspirin $(p=0.006$; $\mathrm{RR}=0.86)$, received $100 \mathrm{mg}$ aspirin $(p<0.00001$; $\mathrm{RR}=0.34$ ). According to the comparison of different low dose of aspirin, perhaps $100 \mathrm{mg}$ is the best dose to prevent preterm birth.

Conclusion: The effect of aspirin on PTB was significantly related to the gestational age and dose of intervention. According to our analysis of the literature, aspirin is recommended to prevent PTB at a dose of $100 \mathrm{mg}$ and before 20 weeks of gestation. PROSPERO registration number: CRD42020148741.

KEYWORDS: Aspirin; Preterm birth; Meta-analysis

\section{INTRODUCTION}

Preterm birth, as a predominant reason of infant mortality, take heavy burden on low-income and middle-income countries $[1,2]$. Meanwhile compared with individuals delivered at term, it associated with the infants' longitudinal adverse effects on health and socioeconomic outcomes [3-5]. Although improvements in neonatal care have resulted in improved survival in high-income countries and some low-income countries regions6, this care is often limited or unavailable in regions with the highest burden of mortality. Therefore, seek for effective strategies for the prevention of PTB have been proved urgent. Trials of low dose aspirin (60-150 mg a day), prevent preeclampsia, also suggest that women receiving aspirin have a reduced occurrence of PTB7-11. Researches point out that this effect might be enhanced when low-dose aspirin is started before 16 weeks of gestation8. Some trials were designed to test the effect of low- dose aspirin in reduce the incidence of
Quick Response Code:

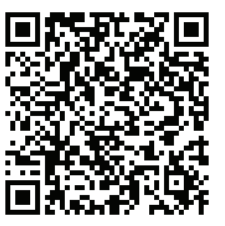

Address for correspondence: Fuchuan Wang, Department of Obstetrics and Gynecology, Beijing Di-Tan Hospital, Capital Medical University, address: China

Received: August 17, $2020 \quad$ Published: August 28, 2020

How to cite this article: Fuchuan W, Wei Y, Li L, Mingfang Z, Xiuzhen C, Juanjuan Y, Xuemei L. Low-dose Aspirin for Preventing Preterm Birth: A Meta-Analysis. 2020 - 2(4) OAJBS.ID.000212. DOI: $10.38125 / \mathrm{OAJBS} .000212$ 
PTB. But trials are different in doses of aspirin and intervening gestational weeks. We summarised the findings of primary and secondary PTB prevention interventions, in order to explore the best dose of aspirin to prevent PTB and the most appropriate start of intervention gestational weeks.

\section{MATERIALS AND METHODS}

This meta-analysis was reported in accordance with the Preferred Reporting Items for Systematic Reviews and MetaAnalyses (PRISMA) guidelines. The systematic review protocol was registered in PROSPERO (CRD42020148741). Ethical approval was not required.

\section{Criteria}

We used three keywords and synonyms to perform systematic literature searches of PubMed, Embase, the Cochrane Central Register of Controlled Trials, the Cochrane library and Clinical Trials gov. Neither filters nor language or location restrictions were applied to any of the searches. All references from the included trials were manually searched. All randomized trials assessing women treated with aspirin versus placebo, published between 2003 and March 2020 and written in English were included. We accepted that PTB was diagnosed as delivery before 37 weeks when it followed pre-labor PROM or spontaneous labor with intact membranes. Trials mainly focused on PTB, we assessed the time between 20 weeks of gestation to 1) spontaneous PTB, 2) iatrogenic PTB, 3) any PTB by performing a Kaplan-Meier analysis for those women with PTB.

\section{Study Selection, Data Extraction}

Each study was independently evaluated by two reviewers according as the inclusion/exclusion criteria, the disputes was resolved by a third reviewer and the team settled disagreements through discussion. The title and abstract were preliminarily selected then followed by a detailed screening of the full paper. Each step in the review process are recorded in the PRISMA flow diagram (Figure 1). Two authors independently extracted data from eligible studies. The outcome measure was the incidence of prematurity.

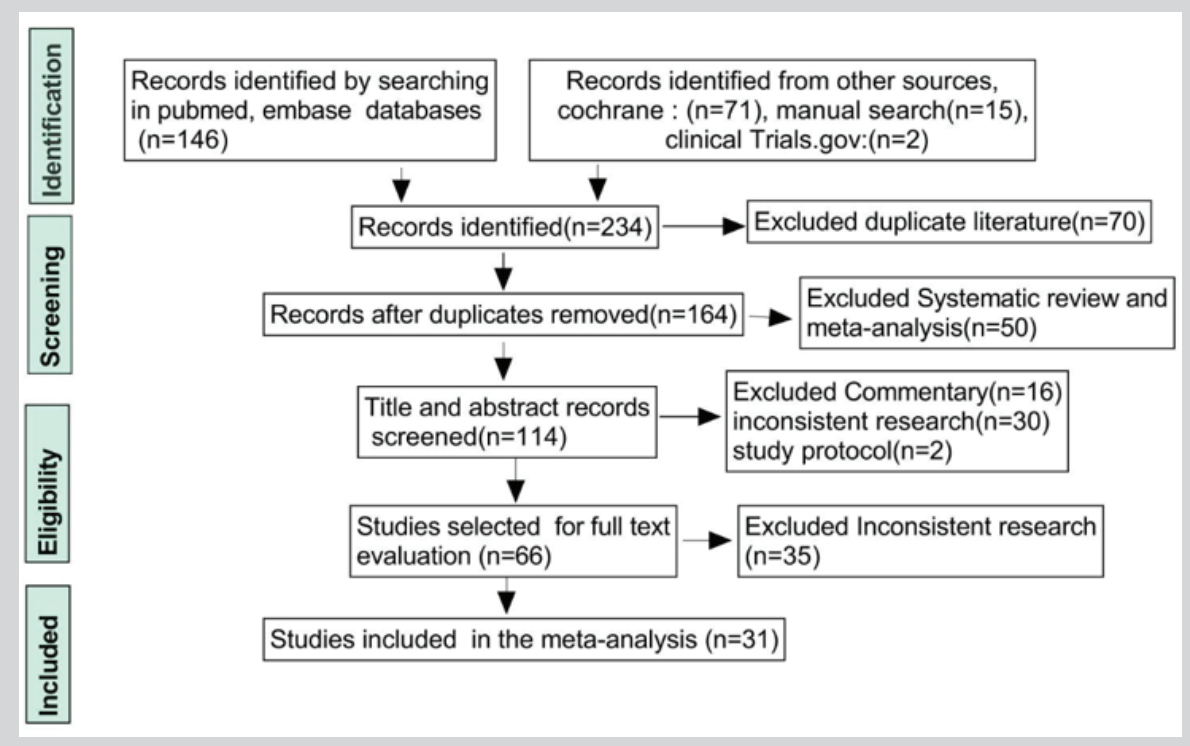

Figure 1: PRISMA flow diagram.

\section{Quality Assessment of Included Studies (Risk of Bias in Individual Studies)}

Two authors assessed the quality and validity of each study independently using the Cochrane Collaboration tool for assessing risk of bias, which included seven categories: (1) Random sequence generation (selection bias); (2) Allocation concealment (selection bias); (3) Blinding of participants and personnel (performance bias); (4) Blinding of outcome assessment (detection bias); (5) Incomplete outcome data (attrition bias); (6) Selective reporting (reporting bias) and (7) Other bias. We classified these categories as low risk of bias, uncertain risk of bias or high risk of bias.

\section{Statistical Analysis}

The meta-analysis was performed using RevMan version 5.312. Heterogeneity was tested with the I 2 statistic (I $2 \geq 50 \%$ or $p<0.1$ represented heterogeneity) and leave-one-out testing for individual studies were used for sensitivity analyses. Publication bias were assessed by funnel plots. We calculated the risk ratio (RR) for dichotomous data with $95 \%$ confidence intervals (CIs). $P<0.05$ indicated statistical significance.

\section{RESULTS}

\section{Study Selection}

We retrieved two hundred and nineteen potentially relevant papers by electronic searching of the specified databases, and fifteen further studies were found via additional manual searching. A total of one hundred and sixty-four records were retained after duplicates were removed. Then we removed fifty systematic reviews and meta-analyses, thirty studies with inconsistent research content, sixteen conference reviews and commentaries, and two study protocol after assessing the titles/abstracts, sixtysix trials received further full text assessment. Thirty-five studies were eliminated after review of the full text, and a total of thirtyone studies, representing 51949 pregnancies, were suitable for the meta-analysis and remained eligible for inclusion in this synthesis [11,13-42]. A total of 27235 patients were treated with aspirin 
and 27214 patients received placebo. Figure 1 shows the selection process of the studies. Seven standards were assessed according to risk of bias, and all studies were included on account of the low risk of bias. The risk-of-bias assessment is described in Figure $2 \&$ Figure 3.

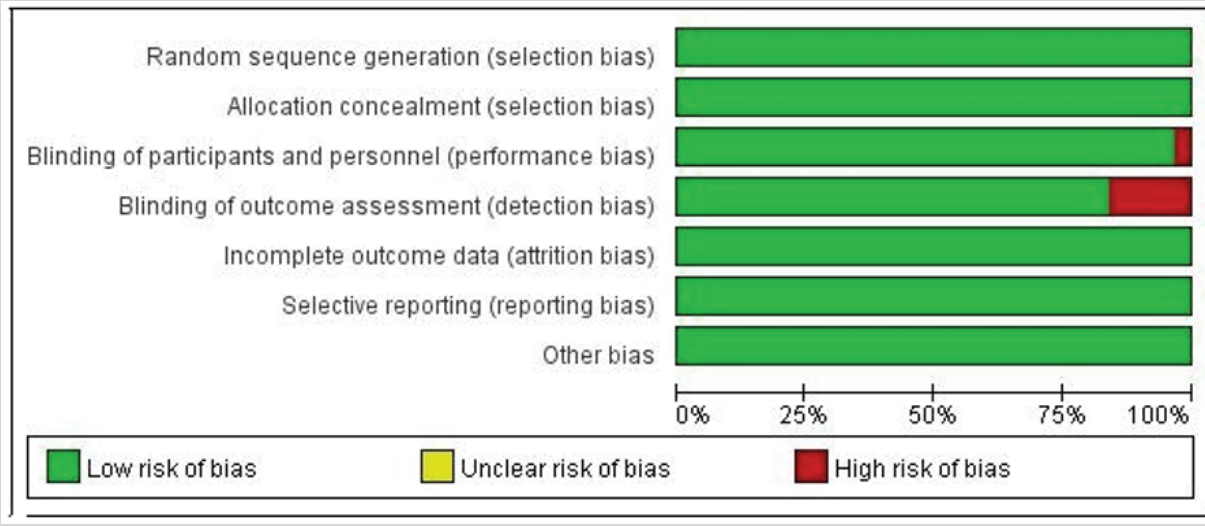

Figure 2: Risk-of-bias graph.

Outcomes of low-dose aspirin for preventing PTB. In all included studies there were no significant differences in maternal age, body mass index or gestational age before treatment. First of all, all studies ( $n=51949$ patients) were included in the analysis of the incidence of PTB after randomization. The result show that there is heterogeneity among the studies $(P=0.02, I 2=37 \%)$. Sensitivity analysis suggested that the heterogeneity was because the incidence of PTB in one study Abdelouahab [14] was less obvious than in all the other studies, so after the study was removed, the results showed that the remaining thirty articles had no heterogeneity $(\mathrm{I} 2=23 \%,<50 \% ; P=0.13,>0.1)$ and that aspirin could significantly reduce the incidence of PTB (RR $=0.89,95 \%$ CI [0.85-0.94], $P<0.00001$ ) compared with placebo (Figure 4). Outcomes if aspirin was commenced at different weeks gestation on PTB. According to the different gestational weeks of aspirin intervention, we further divided all studies into groups to study whether the influence of different intervention gestational weeks on PTB was different. Seven studies, including 14448 women who received low-dose aspirin or placebo from early gestation (gestation $\leq 13$ weeks), were analysed in the analysis of the incidence of PTB. Aspirin reduced the risk of PTB ( $p=0.0006 ; \mathrm{RR}=0.83 ; 95 \%$ CI $[0.75$, 0.92]; I2=34\%).

Seventeen studies ( $n=19419$ patients), which reported women received low-dose aspirin or placebo $\leq 20$ weeks gestation, were analysed the incidence of PTB. There was a significant difference between the two groups ( $p<0.00001$; RR=0.83; $95 \%$ CI $[0.76,0.90]$; $\mathrm{I} 2=32 \%$ ).

Twenty-two studies ( $n=38591$ patients), contained low-dose aspirin commenced $>13$ gestation weeks, were included in the analysis of $\mathrm{PTB}(p=0.001 ; \mathrm{RR}=0.91 ; 95 \% \mathrm{CI}[0.87,0.96] ; \mathrm{I} 2=20 \%)$. Seven studies ( $n=4107$ patients), received low-dose aspirin or placebo from 20 gestation weeks, were included in the analysis of PTB ( $p=0.50 ; \mathrm{RR}=0.94 ; 95 \%$ CI $[0.8,1.12]$; I2=20\%); (Figure 5).

\section{Dose}

Outcomes if Aspirin was Commenced at Different

We further stratified all studies into subgroups, according to the different dose of aspirin, to explore whether the influence of different dose of aspirin on PTB. Eleven studies ( $n=34253$ patients), including study on $60 \mathrm{mg}$ aspirin, were analysed the incidence of
PTB ( $p=0.007 ; \mathrm{RR}=0.92 ; 95 \%$ CI $[0.87,0.98]$; I2=0\%). Five studies ( $n=4470$ patients), which reported women received $75 \mathrm{mg}$ aspirin, were analysed the incidence of PTB. There is heterogeneity among the studies $(P=0.05, \mathrm{I} 2=59 \%)$, and sensitivity analysis revealed that the heterogeneity came from one study Rotchell [42], the results, after the study was removed, showed that the remaining four articles had no heterogeneity $(\mathrm{I} 2=39 \%,<50 \% ; P=0.17,>0.1)$ and the incidence of PTB (RR=0.52, 95\% CI [0.32-0.84], $P=0.008)$ compared with placebo was decreased. Six studies ( $n=12588$ patients), which contained women received about $80 \mathrm{mg}$ aspirin, were analysed the incidence of PTB. There was a significant difference between the two groups ( $p=0.006$; RR=0.86; 95\% CI [0.77, 0.96]; I2=0\%). Eight studies ( $n=964$ patients), which reported women received $100 \mathrm{mg}$ aspirin, were included in the analysis of $\operatorname{PTB}(p<0.00001 ; \mathrm{RR}=0.34$; 95\% CI [0.22, 0.54]; I2=8\%); (Figure 6) .

\section{DISCUSSION}

Aspirin is increasingly used to prevent PTB. Our study, based on thirty-one studies ( $n=51949$ participants), was aimed to evaluate the effect of aspirin on PTB11,13-42. The result shows that low-dose aspirin in pregnant women reduces the RR of PTB by approximately $11 \%$. This is similar to recent MK Hoffman's research, their study suggested that low-dose aspirin reduced 11\% delivery before 37 weeks' gestation than women on a placebo regimen. Additionally, low-dose aspirin, no increase in serious adverse events in mothers or infants, can reduce $25 \%$ PTB before 34 weeks, and perinatal mortality was decreased by $14 \%$ compared with the placebo group [30]. Although aspirin can prevent PTB, however the dose of aspirin and the gestational week of intervention were different in each randomized controlled study. Therefore, to explore the effect of different intervention doses and weeks of aspirin on PTB in order to optimize the program of aspirin prevention of PTB is very important. So, we further evaluated the effect of different doses of aspirin in different gestational weeks on PTB. Analysis was stratified according to gestation at commencement of treatment, the results revealed that there is no difference about the effect of low-dose aspirin commenced $\leq 13$ weeks gestation compared with commenced $\leq 20$ weeks gestation, both can reduce the rate of PTB by $17 \%$, it's also consistent with a new study30. But if aspirin commenced $>20$ gestation weeks, the prevention of PTB is disappearing. Because the dose of aspirin intervention varied from 
study to study. We further evaluated the effect of different doses of aspirin on PTB. According to the comparison of different low-dose of aspirin, the results show that $100 \mathrm{mg}$ aspirin has the best effect in preventing PTB compared with other low-dose aspirin, and
$100 \mathrm{mg}$ aspirin can reduce the incidence of premature birth by $66 \%$, however the intervention dose of $60 \mathrm{mg}$ aspirin can only reduce the PTB rate by $17 \%$.

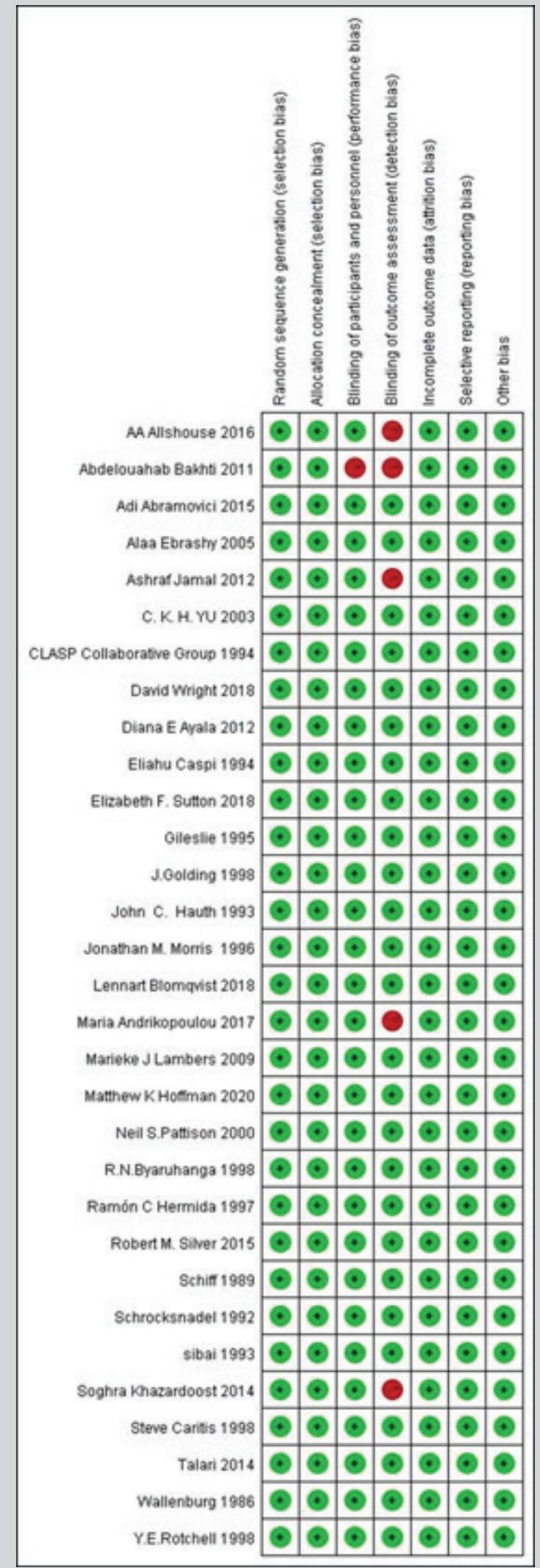

Figure 3: Risk-of-bias and methodological quality summary, containing each methodological quality item for each included study. +: low risk; -: high risk. 

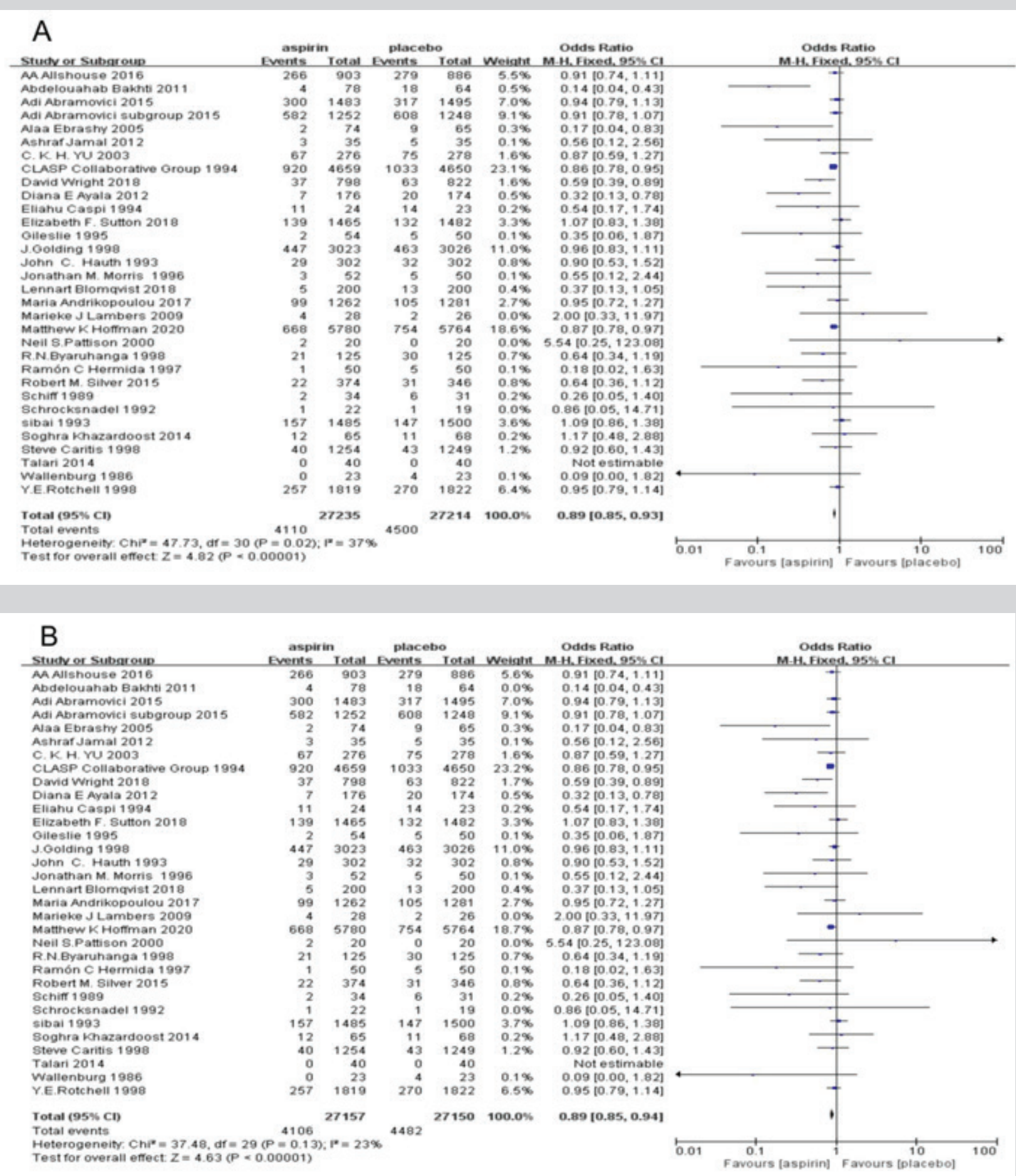

Figure 4: Forest plots for PTB. (A) Thirty-one studies were included and the results show that there is great heterogeneity among the studies $(P=0.02,12=37 \%)$; (B) Sensitivity analysis showed that the heterogeneity was because one study (Abdelouahab Bakhti 2011), after the study was removed, the results had no heterogeneity $(12=23 \%, \quad<50 \% ; P=0.13$, $>0.1$ ). MD: Mean difference; $\mathrm{Cl}$ : Confidence interval; RR: Risk ratio. 


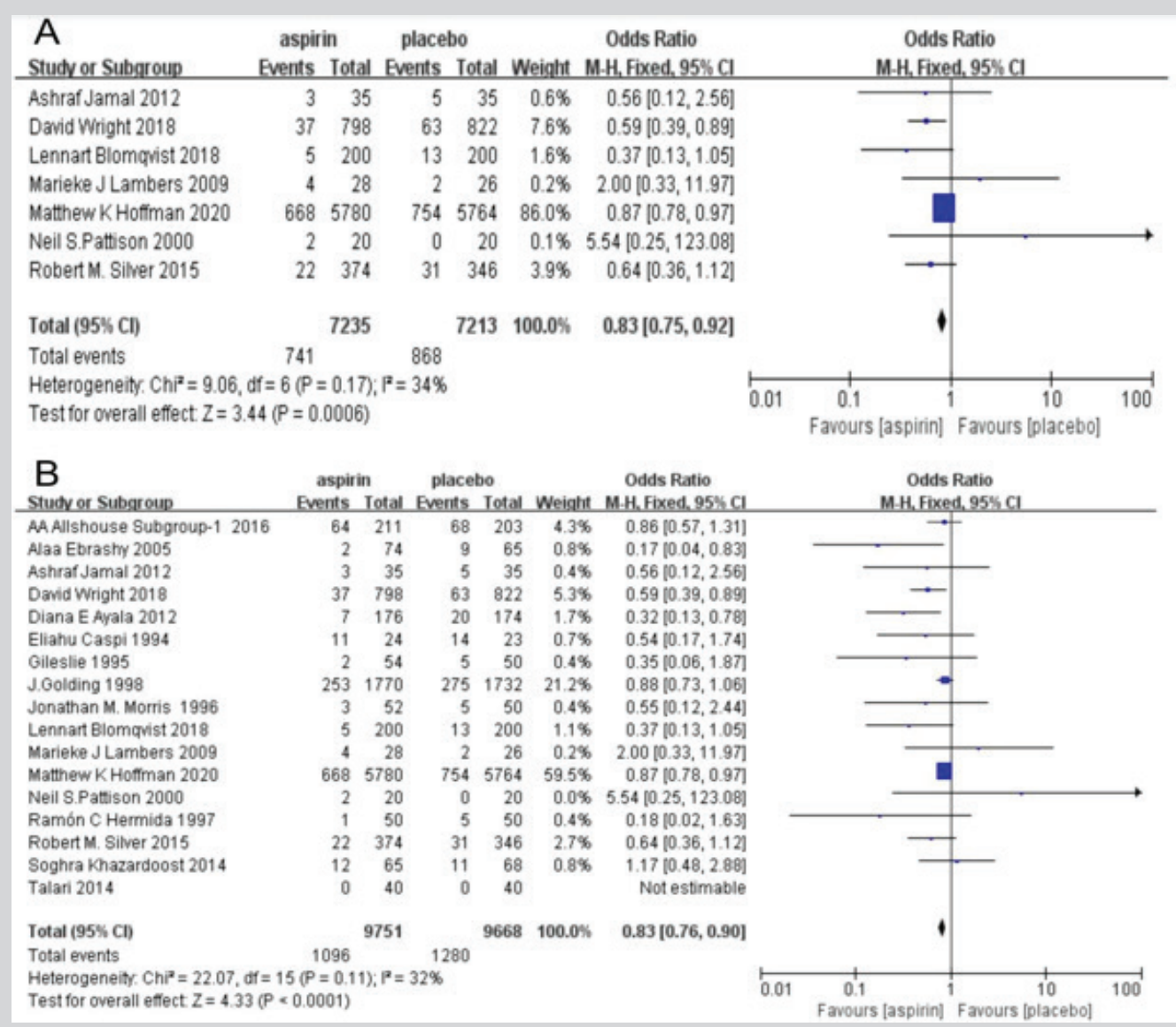

\section{C}

stucver subaroun

MA Alshouse Subgroup:2 2016

Adi Abramonici 2015

Adi Abramonci subgroup 2015

Alaa Ebrashy 2005

CLASP Collaborative Oroup 1994

Diana E Ayala 2012

Elizabeth F. Sumton 2018

Olleslie 1995

J.Golding 1998
Jonathan M. Morns 1996
Maria Andrikopoulou 2017

Ramón C Hermida 1997

Ramirn 1989

Schrocksnadel 1992

sibai 1993
Soghra Khazardoost 2014

Steve Caritis 1998

Wallenburg 1986

Y.E. Rotchell 1998

Totat (9Ss CI)
Total events

Total ewents

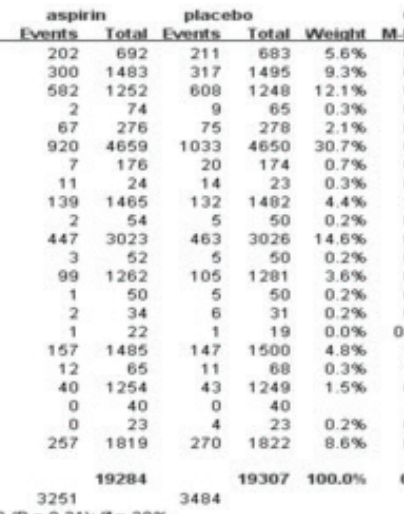

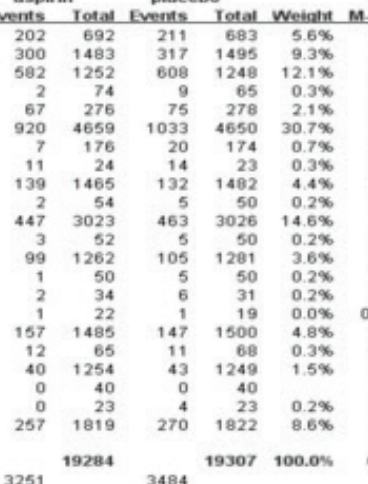

Test for overall effect $Z=3.24(P=0.001)$

Odds Ratio
M. Odds Ratio
M. Fixed. $95 \%$ CI $95 \%$ C

$0.94[0.79,1.13]$

$0.17[0.04,0.83)$

$0.87[0.59,1.27]$

$0.86[0.78,0.95$
$0.32[0.13,0.78$

$0.5410 .17,1.74$

$1.07[0.83,1.301$
$0.35[0.06,1.87]$

$0.9650 .83,1.11$

$0.55[0.12,2.44$
$0.95[0.72,1.27$

$0.26[0.05,1.40$

$0.8610 .05,14.71$

$1.0910 .86,1.38$
$1.1710 .48,2.809$

Not estimabie

$0.0910 .00,1.821$

0.91 [0.87, 0.96]

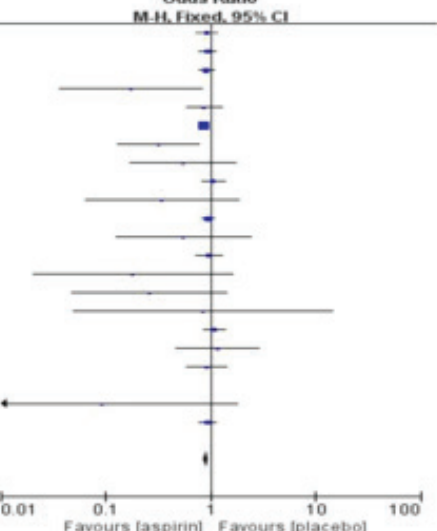

D $\quad$ aspirin placebo Odds Ratio $\quad$ Odds Ratio

Studv or Subgroup Events Total Events Total Weight M.H. Fixed, $95 \% \mathrm{Cl} \quad$ M.H. Fixed, $95 \% \mathrm{Kl}$

\begin{tabular}{lllllll}
\hline C.K.H.YU 2003 & 67 & 276 & 75 & 278 & $20.3 \%$ & 0.87 \\
{$[0.59,1.27]$}
\end{tabular}

$\begin{array}{lllllll}\text { J.Golding 1998 } \quad 194 & 1253 & 188 & 1294 & 56.2 \% & 1.08[0.87,1.34]\end{array}$

$\begin{array}{lllllll}\text { John C. Hauth } 1993 & 29 & 302 & 32 & 302 & 10.4 \% & 0.90[0.53,1.52]\end{array}$

$\begin{array}{lllllll}\text { R.N.Byaruhanga } 1998 & 21 & 125 & 30 & 125 & 9.0 \% & 0.64[0.34,1.19]\end{array}$

$\begin{array}{lllllll}\text { Schiff } 1989 & 2 & 34 & 6 & 31 & 2.1 \% & 0.26[0.05,1.40]\end{array}$

$\begin{array}{lllllll}\text { Schrocksnadel 1992 } & 1 & 22 & 1 & 19 & 0.4 \% & 0.86[0.05,14.71]\end{array}$

$\begin{array}{lllllll}\text { Wallenburg } 1986 & 0 & 23 & 4 & 23 & 1.6 \% & 0.09[0.00,1.82]\end{array}$

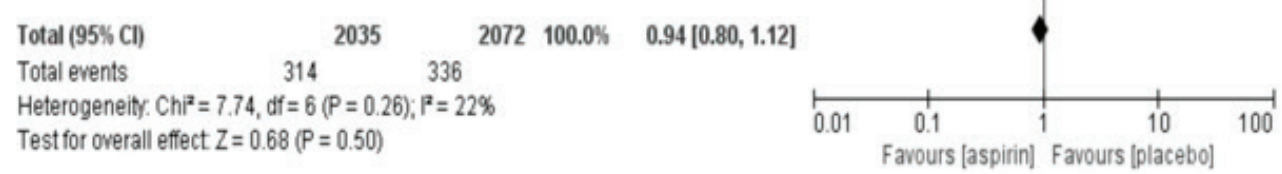

Figure 5: Forest plots for aspirin was commenced at different gestation weeks. (A) Low-dose aspirin or placebo was commenced $\leq 13$ gestation weeks; (B) Commenced $\leq 20$ gestation weeks; (C) Commenced >13 gestation weeks; (D) Commenced >20 gestation weeks. MD: Mean difference; Cl: Confidence interval; RR: Risk ratio. 


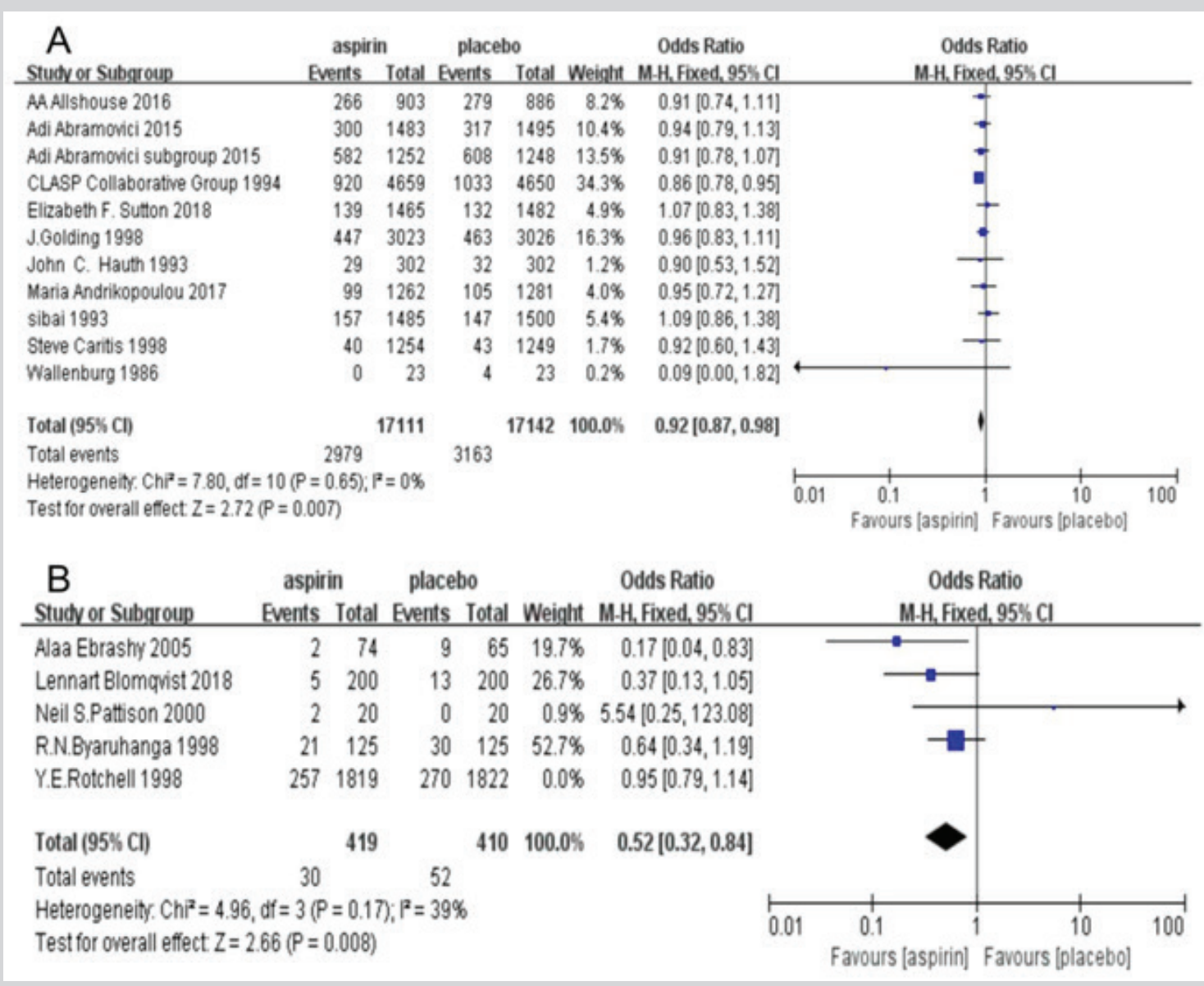

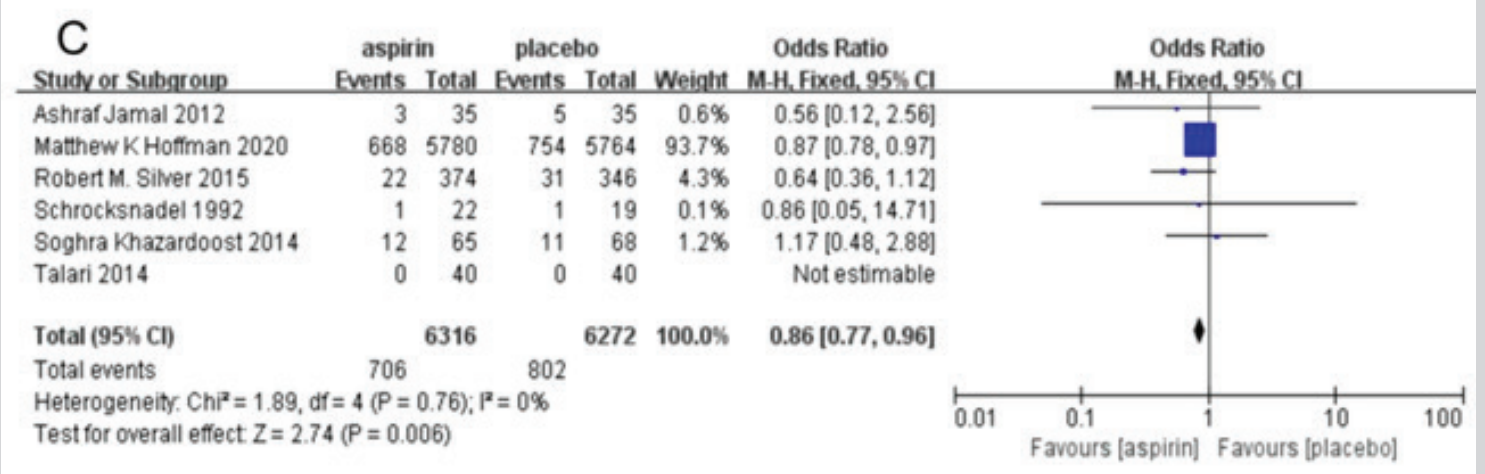

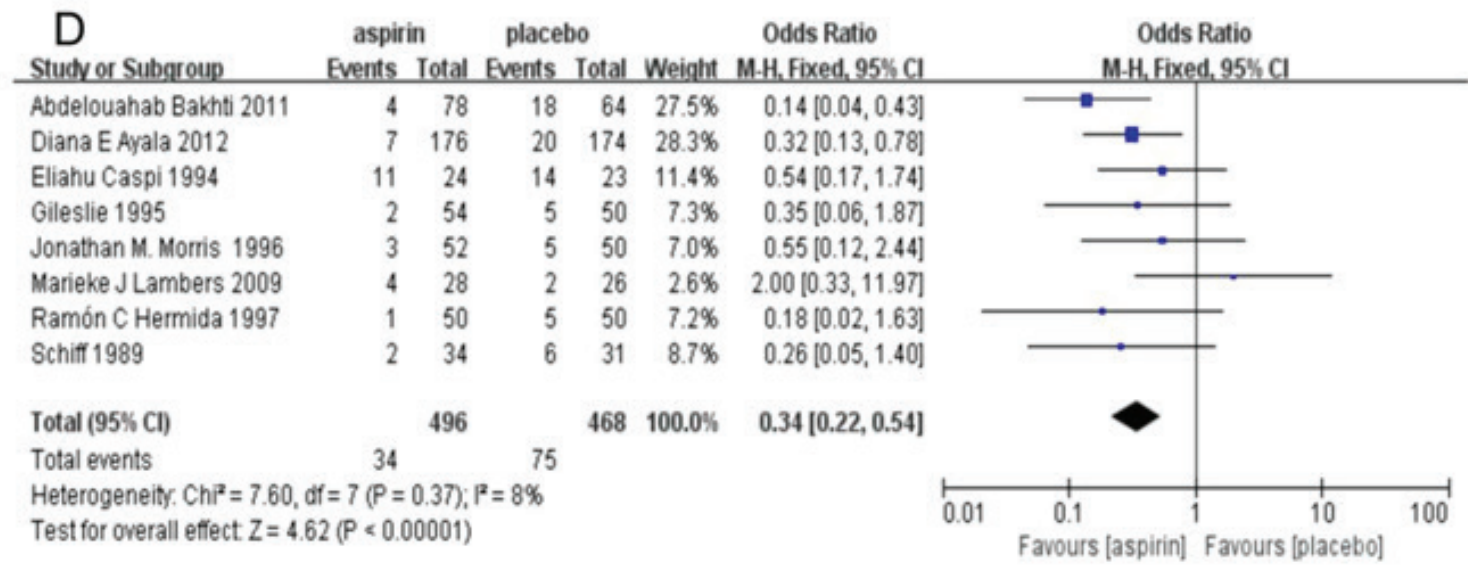

Figure 6: Forest plots for aspirin was commenced at different dose. (A) The dose of $60 \mathrm{mg}$ aspirin; (B) The dose is about $75 \mathrm{mg}$ aspirin; (C) The dose is about $80 \mathrm{mg}$ aspirin; (D) The treatment dose is $100 \mathrm{mg}$ of aspirin; MD: Mean difference; $\mathrm{Cl}$ : Confidence interval; RR: Risk ratio. 
The therapeutic effect of aspirin for PTB has been uniformly recognized at home and abroad. Therefore, it is very important to explore the best intervention week and the most appropriate intervention dose of aspirin. According to our study, different gestational weeks of aspirin intervention within 20 weeks of pregnancy have little effect on PTB, so the intervention time can be appropriately delayed within 20 weeks of pregnancy. However, different doses of aspirin at the beginning of intervention have great influence on the effect of PTB, these findings support the notion that it is recommended to choose $100 \mathrm{mg}$ aspirin before 20 weeks of pregnancy.

However, limitations of our study should be noted. Due to the lack of research on aspirin prevention of PTB, the existing research data are not enough to further study the difference effect on PTB of different intervention weeks before 20 weeks. In the future study, we will further refine the effect of different aspirin start intervention weeks on the prevention of PTB, so as to choose the best start intervention weeks.

\section{CONCLUSION}

In summary, our results show that low-dose aspirin can significantly reduce preterm birth. This provides support for the more conventional use of low-dose aspirin as a treatment strategy that can improve the management of reduce preterm.

\section{AUTHORS CONTRIBUTION}

Fuchuan Wang conceived the study. Wei Yi, Li Li, and Mingfang Zhou selected studies for inclusion and abstracted data. Fuchuan Wang and Xiuzhen Cao performed the statistical analyses. Wei Yi and Juanjuan Yao interpreted the data. Fuchuan Wang, Juanjuan Yao and Xuemei Liu wrote the first draft.

\section{STATEMENT OF ETHICS}

All analyses were based on the published studies, and thus, no patient consent was required.

\section{REFERENCES}

1. Blencowe H, Cousens S, Oestergaard MZ (2012) National, regional, and worldwide estimates of preterm birth rates in the year 2010 with time trends since 1990 for selected countries: a systematic analysis and implications. Lancet 379: 2162-2172.

2. Liu L, Oza S, Hogan D (2016) Global, regional, and national causes of under-5 mortality in 2000- 15: an updated systematic analysis with implications for the sustainable development goals. Lancet 388: 3027 3035 .

3. Moster D, Lie RT, Markestad T (2008) Long-term medical and social consequences of preterm birth. N Engl J Med 359(3): 262-273.

4. Bilsteen JF, Taylor-Robinson D, Børch K, Strandberg-Larsen K, Nybo Andersen AM (2018) Gestational age and socioeconomic achievements in young adulthood: a Danish population-based study. JAMA Netw Open 1(8): e186085.

5. Blencowe H, Lee AC, Cousens S (2013) Preterm birth-associated neurodevelopmental impairment estimates at regional and global levels for 2010. Pediatr Res 74 (suppl 1): 17-34.

6. Liu L, Johnson HL, Cousens S (2012) For the child health epidemiology reference group of WHO and UNICEF. Global, regional and national causes of child mortality: An updated systematic analysis for 2010 with time trends since 2000. Lancet 379(9832): 2151-2161.

7. Henderson J, Whitlock E, O'Connor E, Senger C, Thompson J, et al. (2014) Low-dose aspirin for the prevention of morbidity and mortality from pre-eclampsia. A systematic evidence reviews for the US Preventive Services Task Force. Agency for Healthcare Research and Quality. Evidence syntheses, no. 112. Rockville, MD, USA.
8. Roberge S, Nicolaides KH, Demers S, Villa P, Bujold E (2013) Prevention of perinatal death and adverse perinatal outcome using low-dose aspirin: a meta-analysis. Ultrasound Obstet Gynecol 41(5): 491-499.

9. Askie LM, Duley L, Henderson-Smart DJ, Stewart LA (2007) Antiplatelet agents for prevention of pre- eclampsia: a meta-analysis of individual patient data. Lancet 369(9575): 1791-1798.

10. Silver RM, Ahrens K, Wong LF (2015) Low-dose aspirin and preterm birth: a randomized controlled trial. Obstet Gynecol 125(4): 876-884.

11. Andrikopoulou M, Purisch SE, Handal-Orefice R, Gyamfi-Bannerman C (2018) Low-dose aspirin is associated with reduced spontaneous preterm birth in nulliparous women. Am J Obstet Gynecol 219: e1-e6.

12. (2018) R Core Team: A language and environment for statistical computing. Version 3.5.1. Vienna: R Foundation for Statistical Computing.

13. Allshouse AA, Jessel RH, Heyborne KD (2016) The impact of low-dose aspirin on preterm birth: secondary analysis of a randomized controlled trial. J Perinatology 36(6): 427-431.

14. Abdelouahab B, Daniel V (2011) Prevention of gravidic endothelial hypertension by aspirin treatment administered from the $8^{\text {th }}$ week of gestation. Hypertension Research 34(10): 1116-1120.

15. Adi A, Victoria J, Luisa W (2015) Low-dose aspirin, smoking status and the risk of spontaneous preterm birth. Am J Perinatol 32(5): 445-450.

16. Alaa E, Magdy I, Ayman M (2005) Usefulness of aspirin therapy in highrisk pregnant women with abnormal uterine artery doppler ultrasound at 14-16 weeks pregnancy: Randomized controlled clinical trial. Croat Med J 46(5): 826-831.

17. Ashraf J, Forozan M, Ashraf Al-Yasin (2012) Evaluation of the effect of metformin and aspirin on utero placental circulation of pregnant women with PCOS. Iran J Reprod Med 10(3): 265-270.

18. Yu CKH, Papageorghiou AT, Parra M, Palma DR, Nicolaides KH (2003) Randomized controlled trial using low-dose aspirin in the prevention of pre-eclampsia in women with abnormal uterine artery Doppler at 23 weeks' gestation. Ultrasound Obstet Gynecol 22(3): 233-239.

19. (1994) CLASP (Collaborative Low-dose Aspirin Study in Pregnancy) Collaborative Group. CLASP: a randomised trial of low-dose aspirin for the prevention and treatment of pre-eclampsia among 9364 pregnant women. Lancet 343: 619-29.

20. Wright D, Nicolaides KH (2019) Aspirin delays the development of preeclampsia. Am J Obstet Gynecol 220(6): E1-E6.

21. Diana EA, Rafael U, Ramón CH (2013) Chronotherapy with low-dose aspirin for prevention of complications in pregnancy. Chronobiol Int 30(1-2): 260-279.

22. Caspi E, Raziel A, Sherman D (1994) Prevention of pregnancy-induced hypertension in twins by early administration of low-dose aspirin: A preliminary report. Am J Reprod Immunol 31(1): 19-24.

23. Elizabeth FS, Alisse H, Steve NC (2018) Maternal outcomes associated with lower range stage 1 Hypertension. Obstet Gynecol 132: 843-849.

24. Leslie GI, Gallery ED, Arnold JD (1995) Neonatal outcome in a randomized, controlled trial of low-dose aspirin in high-risk pregnancies. J Paediatr Child Health 31: 549-552.

25. Golding J (1998) A randomised trial of low dose aspirin for primiparae in pregnancy. British Journal of Obstetrics and Gynaecology 105(3): 293-299.

26. John CH, Robert LG (1993) Low-dose aspirin therapy to prevent preeclampsia. Am J Obstet Gynecol 168(4): 1083-1091.

27. Morris JM, Fay RA, Ellwood DA (1996) Randomized controlled trial of aspirin in patients with abnormal uterine artery blood flow. Obstet Gynecol 87(1): 74-78.

28. Blomqvist L, Hellgren M, Strandell A (2018) Acetylsalicylic acid does not prevent first trimester unexplained recurrent pregnancy loss: a randomized controlled trial. Acta Obstet Gynecol Scand 97(11): 13651372. 
29. Marieke JL, Groeneveld Els, Diederik AH (2009) Lower incidence of hypertensive complications during pregnancy in patients treated with low-dose aspirin during in vitro fertilization and early pregnancy. Human Reproduction 24(10): 2447-2450.

30. Matthew KH, Shivaprasad SG, Bhalachandra SK (2020) Low-dose aspirin for the prevention of preterm delivery in nulliparous women with a singleton pregnancy (ASPIRIN): A randomised, double-blind, placebocontrolled trial. Lancet 395: 285-293.

31. Neil SP, Lawrence WC, Mary B (2000) Does aspirin have a role in improving pregnancy outcome for women with the antiphospholipid syndrome? A randomized controlled trial. Am J Obstet Gynecol 183(4): 1008-10012.

32. Byaruhanga RN, Chipato T, Rusakaniko S (1998) A randomized controlled trial of low-dose aspirin in women at risk from pre-eclampsia. International Journal of Gynecology \& Obstetrics 60(2): 129-135.

33. Robert MS, Katherine A, Luchin FW (2015) Low-dose aspirin and preterm birth. Obstet Gynecol. 125(4): 876-84.

34. Schiff E, Peleg E, Goldenberg M (1989) The use of aspirin to prevent pregnancy-induced hypertension and lower the ratio of thromboxane A2 to prostacyclin in relatively high-risk pregnancies. N Engl J Med 321(6): 351-356.

35. Schrocksnadel HSB, Alge A, Stechel-Berger G (1992) Low-dose aspirin in primigravidae with positive rollover test. Gynecol Obstet Invest 34: 146-150.
36. Sibai BM, Caritis SN, Thom E (1993) Prevention of preeclampsia with low-dose aspirin in healthy, nulliparous pregnant women. The national institute of child health and human development network of maternalfetal medicine units. N Engl J Med 329(17): 1213-1218.

37. Soghra K, Sanaz M, Sedigheh B (2014) Effect of aspirin in prevention of adverse pregnancy outcome in women with elevated alpha-fetoprotein. J Matern Fetal Neonatal Med 27(6): 561-565.

38. Ramón CH, Diana EA, Manuel I (1997) Time-dependent effects of lowdose aspirin administration on blood pressure in pregnant women. Hypertension 30(3): 589-595.

39. Caritis S, Sibai B, Hauth J (1998) Low-dose aspirin to prevent preeclampsia in women at high risk. national institute of child health and human development network of maternal-fetal medicine units. N Engl J Med 338(11): 701-705.

40. Talari H, Mesdaghinia E, Abedzadeh KM (2014) Aspirin and preeclampsia prevention in patients with abnormal uterine artery blood flow. Iran Red Crescent Med J 16: e17175.

41. Wallenburg HC, Dekker GA, Makovitz JW, Rotmans P (1986) Low-dose aspirin prevents pregnancy-induced hypertension and pre-eclampsia in angiotensin-sensitive primigravidae. Lancet 1(8471): 1-3.

42. Rotchell YE, Cruickshank JK, Phillips GM (1998) Barbados low dose aspirin study in pregnancy (BLASP): A randomised trial for the prevention of pre-eclampsia and its complications. British Journal of Obstetrics and Gynaecology 105(3): 286-292. 